\title{
Scleral Buckle Infection Caused by Mycobacterium chelonae: A Case Report
}

This article was published in the following Dove Press journal:

International Medical Case Reports Journal

\author{
Mami Kusaka \\ Yu Kagitani \\ Sachiyo Hama \\ Mihori Kita
}

Department of Ophthalmology, National Hospital Organization Kyoto Medical

Center, Kyoto, Japan
Correspondence: Mihori Kita

Department of Ophthalmology, National

Hospital Organization Kyoto Medical

Center, I-I Mukaihata-Cho, Fukakusa,

Fushimi-Ku, Kyoto-city, Kyoto, 612-8555, Japan

Tel +8I-75-64|-9|6I

Fax +8I-75-643-4325

Email mihorik@kuhp.kyoto-u.ac.jp
Abstract: A 63-year-old female with a history of retinal detachment repair with scleral buckle from 28 years prior presented with exposure of the buckle in her right eye. After removing the buckle and anchoring sutures, the operation field was washed with $0.25 \%$ polyvinyl alcohol-iodine, vancomycin, and ceftazidime. Cultures revealed Mycobacterium chelonae. Systemic meropenem was discontinued on postoperative day 5. The retina remained attached, and there was no recurrence over a 1-year follow-up. We report a case of NTM buckle infection that responded well to thorough washings with polyvinyl alcoholiodine and antibiotic solutions instead of using a prolonged course of systemic antibiotics.

Keywords: scleral buckle, infection, Mycobacterium chelonae, nontuberculous mycobacteria, NTM, polyvinyl alcohol-iodine, artificial device

\section{Introduction}

Scleral buckle infection is rare, with a reported incidence varying from $0.5 \%$ to $5.6 \%$. ${ }^{1}$ However, this infection can potentially lead to severe complications such as endophthalmitis, panophthalmitis, and orbital cellulitis. ${ }^{2,3}$ Nontuberculosis mycobacterium infections are typically hard to manage due to poor drug penetration and a slow response to treatment. ${ }^{4}$ As a result, prolonged administration of systemic antibiotics is often necessary.

This report presents a case of Mycobacterium chelonae (M.chelonae) scleral buckle infection in which systemic antibiotics were successfully stopped a few days after removal of the buckle followed by thorough washings with $0.25 \%$ polyvinyl alcohol-iodine and antibiotics.

\section{Case Report}

This study was approved by the Ethics Committee of the National Hospital Organization Kyoto Medical Center, and all procedures involved adhered to the tenets of the Declaration of Helsinki. Written informed consent for the procedure and participation in the study including case details, images, and videos published was obtained from the patient after receiving an explanation of the therapeutic procedures.

A healthy 63-year-old female with a history of retinal detachment repair of her right eye including four scleral buckling surgeries 28 years ago was referred to our clinic. She had been suffering from a discharge in her right eye for 2 weeks. Slitlamp examination revealed conjunctival injection of the right eye without any sign of iridocyclitis or keratitis, and exposure of the scleral buckle (Figure 1). She had 


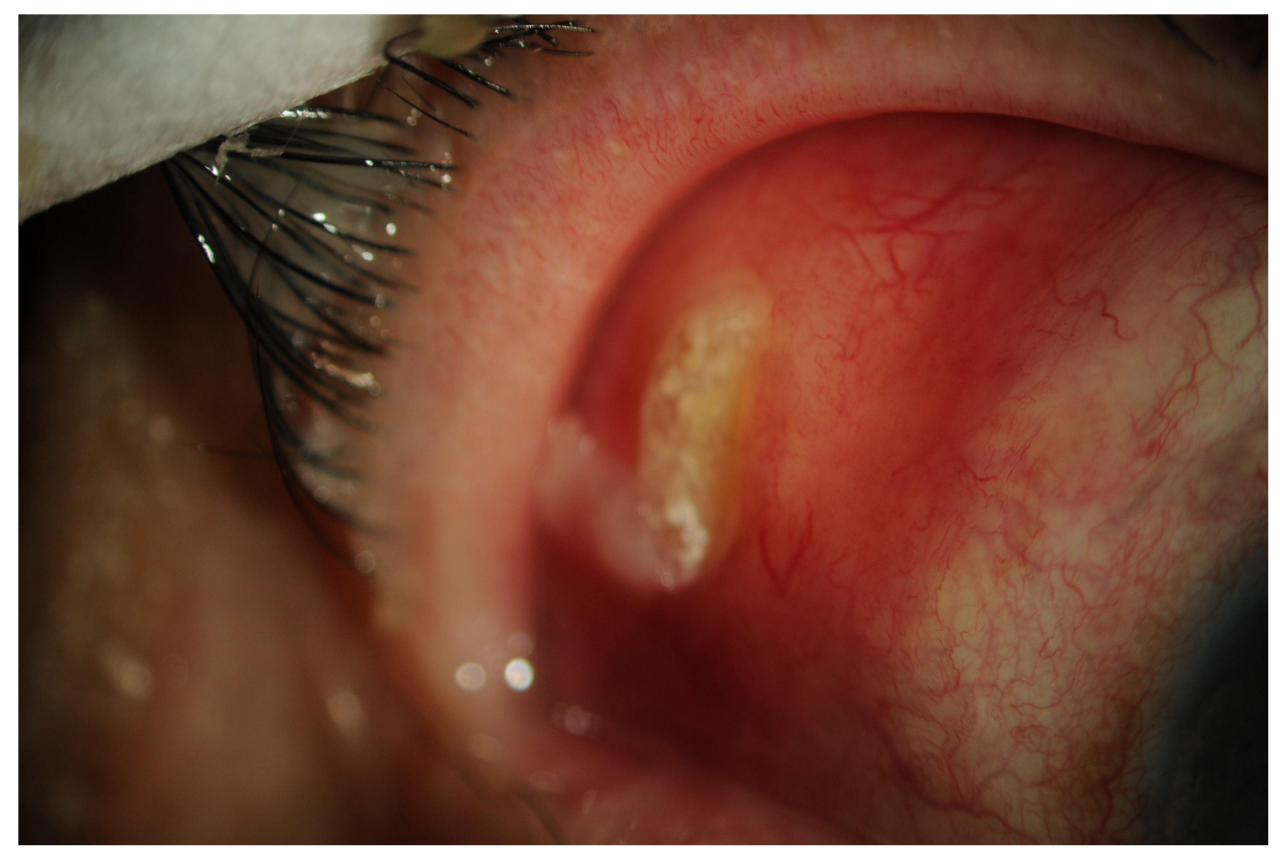

Figure I Exposed sponge buckle at the superior-temporal area at $8 \mathrm{~mm}$ from the limbus, and injection of conjunctiva were observed in her right eye.

high myopia with an axial length of $33.55 \mathrm{~mm}$ in her right eye. The best-corrected visual acuity was hand motion in the right eye, which did not change after the buckling surgery, and 20/20 in the left eye, for which the axial length was $25.33 \mathrm{~mm}$. Systemic examination, which included a chest X-ray, showed no abnormal findings.

After the culture of the conjunctival swab and subsequently being diagnosed with scleral buckle infection, the patient was started on a topical levofloxacin treatment along with $1000 \mathrm{mg}$ of intravenous meropenem three times a day. On the following day, the encircling sponge buckle and the anchoring sutures were removed. The operation field was then washed with $50 \mathrm{~mL}$ of $0.25 \%$ polyvinyl alcohol-iodine, and the same amount of vancomycin $(10 \mathrm{mg} / \mathrm{mL})$ and ceftazidime $(20 \mathrm{mg} / \mathrm{mL})$ through the conjunctival tunnel that was formed during the buckle removal. (Supplementary Video 1) During the final step of the surgery, a subconjunctival injection of vancomycin and ceftazidime was performed. After the conjunctival injection and discharge disappeared on postoperative day 5 , the systemic antibiotic was then discontinued. The retina remained attached, and there has been no recurrence of the inflammation over the 1-year follow-up.

On postoperative day 7, all of the culture results for the conjunctiva swab obtained at the first visit, and for the removed buckle and sutures identified the presence of $M$. chelonae (Gaffky scale 2). On postoperative days 6 and 8, there was no longer any growth found for the subsequent cultures of the conjunctival swab.

\section{Discussion}

One of the postoperative complications of scleral buckling surgery is buckle infection. It was reported that repeated washing of the ocular surface with $0.25 \%$ povidone-iodine during the scleral buckling surgery was able to minimize the ocular surface bacterial contamination at the completion of the procedure. ${ }^{5}$ The usual treatment for buckle infection is the removal of the buckle combined with systemic antibiotic treatment. The most commonly found organisms in these cases are coagulase-negative staphylococci, followed by Mycobacterium chelonae, ${ }^{1}$ which was identified in our current case.

Mycobacterium chelonae belongs to a nontuberculous mycobacteria species (NTM). Although these ubiquitous environmental organisms are found in the soil, dust, and water, they rarely cause infections. However, NTM have been recently recognized as being responsible for the increased morbidity of infections associated with artificial devices such as pacemakers, prosthetic cardiac valves, joint implants, intravenous catheters, and scleral buckle in not only immunosuppressed but also in immunocompetent patients. ${ }^{6-9}$ 
NTM infections are typically challenging to manage due to delayed laboratory identification, poor drug penetration, and slow response to therapy. Moreover, silicone sponge buckle elements can promote the development of a biofilm, which is thought to be responsible for scleral buckle infections even without exposure to the buckle. ${ }^{10,11}$ Fortunately, there could be no biofilm development found in our current case, which would have demonstrated a good response to our therapy.

NTM, especially the rapidly growing mycobacteria (RGM), which include Mycobacterium chelonae, are not sensitive to first-line antituberculous agents. NTM infections in and around the eye, which have been shown to be clinically recalcitrant to treatment, recur after cessation of therapy, and require long-term therapy of antibiotics including amikacin, clarithromycin, and ciprofloxacin. ${ }^{11,12}$

In our current case, we were able to successfully withdraw the use of systemic antibiotics during the early phase of buckle removal, without any recurrence of the inflammation over the 1-year follow-up. Thus, washing of the operation field with $0.25 \%$ polyvinyl alcohol-iodine, along with the administration of antibiotics at the time of the removal of the scleral buckle and sutures as soon as possible after the buckle exposure, can potentially lead to good results in Mycobacterium chelonae buckle infections in immunocompetent patients.

NTM infections are rare, and often require lengthy treatment. We report a case of NTM buckle infection that responded well to thorough washings with $0.25 \%$ polyvinyl alcohol-iodine and antibiotic solutions instead of using a prolonged course of systemic antibiotics.

\section{Patient Consent}

Obtained.

\section{Disclosure}

No conflicting relationship exists for all authors.

\section{References}

1. Chhablani J, Nayak S, Jindal A, et al. Scleral buckle infections: microbiological spectrum and antimicrobial susceptibility. $J$ Ophthalmic Inflamm Infect. 2013;3(1):67. doi:10.1186/18695760-3-67

2. Tsui I. Scleral buckle removal: indications and outcomes. Surv Ophthalmol. 2012;57(3):253-263. doi:10.1016/j.survophthal.2 011.11 .001

3. Nemet AY, Ferencz JR, Segal O, Meshi A. Orbital cellulitis following silicone-sponge scleral buckles. Clin Ophthalmol. 2013;7:2147-2152. doi:10.2147/OPTH.S50321

4. Moorthy RS, Valluri S, Rao NA. Nontuberculous mycobacterial ocular and adnexal infections. Surv Ophthalmol. 2012;57 (3):202-235. doi:10.1016/j.survophthal.2011.10.006

5. Shimada $H$, Nakashizuka $H$, Hattori T, et al. Prophylaxis for acute scleral buckle infection using $0.25 \%$ povidone-iodine ocular surface irrigation during surgery. Int Ophthalmol. 2014;34(2):211-216. doi:10.1007/s10792-013-9816-5

6. Yuan SM. Mycobacterial Endocarditis: a Comprehensive Review. Braz J Cardiovasc Surg. 2015;30:93-103.

7. Hooda A, Pati PK, John B, George PV, Michael JS. Disseminated mycobacterium chelonae infection causing pacemaker lead endocarditis in an immunocompetent host. BMJ Case Rep. 2014;22:bcr2014206042.

8. Roest S, Bax HI, Verkaik NJ, et al. Mycobacterium Chelonae, an 'Atypical' Cause of an LVAD Driveline Infection. Int J Infect Dis. 2020;92:127-129. doi:10.1016/j.ijid.2020.01.002

9. Iordache SD, Daneman N, Axelrod TS. Mycobacterium chelonae infection following silicone arthroplasty of the metacarpophalangeal joints: a case report. Hand. 2009;4(2):129-133. doi:10.1007/s11552008-9138-7

10. Churgin DS, Tran KD, Gregori NZ, Young RC, Alabiad C, Flynn HW Jr. Multi-drug resistant Mycobacterium chelonae scleral buckle infection. Am J Ophthalmol Case Rep. 2018;10:276-278. doi:10.1016/j.ajoc.2018.04.004

11. Shah M, Relhan N, Kuriyan AE, et al. Endophthalmitis caused by nontuberculous mycobacterium: clinical features, antimicrobial susceptibilities, and treatment outcomes. Am J Ophthalmol. 2016;168:150-156. doi:10.1016/j.ajo.2016.03.035

12. Kim AY, Davis AS, Moreau A, Drevets DA, Melendes DP. Management of nontuberculous mycobacterial infections of the eye and orbit: a retrospective case series. Am J Ophthalmol Case Rep. 2020;20:100971. doi:10.1016/j.ajoc.2020.100971

\section{Publish your work in this journal}

The International Medical Case Reports Journal is an international, peer-reviewed open-access journal publishing original case reports from all medical specialties. Previously unpublished medical posters are also accepted relating to any area of clinical or preclinical science. Submissions should not normally exceed 2,000 words or 4 published pages including figures, diagrams and references. The manuscript management system is completely online and includes a very quick and fair peer-review system, which is all easy to use. Visit http://www.dovepress.com/testimonials.php to read real quotes from published authors. 